\section{Trust Modeling for Networked Organizations Using Reputation and Collaboration Estimates}

\author{
Nada Lavrač, Peter Ljubič, Tanja Urbančič, \\ Gregor Papa, Member, IEEE, Mitja Jermol, and Stefan Bollhalter
}

\begin{abstract}
The main motivation for organizations and individuals to collaborate is to enable knowledge and resource sharing in order to effectively fulfill a joint business opportunity. This correspondence focuses on virtual organizations (VOs) and virtual teams (VTs), whose strengths lie in the range of competencies of their members, offered jointly through collaboration. One of the difficulties in VO and VT creation is partner selection using partners' mutual trust as one of the selection criteria. This correspondence provides an analysis of trust relationships based on the principal-agent theory, and proposes an approach to hierarchical multiattribute decisionsupport-based trust estimation applied to a network of collaborating organizations (VO) and a network of collaborating individuals (VT). The correspondence presents two case studies, one using a questionnaire-based approach and the other using automated reputation and collaboration estimation from data gathered by Web crawling.
\end{abstract}

Index Terms-Decision support systems, modeling, networks, visualization.

\section{INTRODUCTION}

W ITH the development of information and communication technologies, numerous formal and informal networks of organizations and individuals started to emerge. Organized networks of organizations and organized networks of individuals are referred to as collaborative networked organizations (CNOs).

- Networks of organizations: In industry, clusters of organizations aim at efficient product development, production, and marketing tasks. Such partner consortia function as networked organizations (NOs) in which cooperation is supported by information and communication technologies. Since the early 1990s, collaboration of European academic and business partners has also increasingly been motivated by numerous EU-funded research and development projects, in which partners share their knowledge and resources to jointly develop new methods, problem-solving protocols, and practical solutions.

- Networks of individuals: As the industrial focus has moved from efficiency to creativity and innovation, human capital is now recognized as an essential competitive advantage of business entities. Many new organizational schemes have also occurred for networks of individuals: virtual communities, communities of practice, professional virtual communities, user forums, etc. These communities bring together individuals of similar interests to communicate and share or exchange information and knowledge.

Among different organizational models for $\mathrm{CNO}$, a virtual enterprise (VE) model, as defined in [1] and [2], has become very popular

Manuscript received December 20, 2005; revised November 7, 2006. This work was supported by the Slovenian Research Agency under Program Knowledge Technologies (2004-2008) and the European 6th FP under integrated project European Collaborative Networked Organizations Leadership Initiative (ECOLEAD, 2004-2008). This paper was recommended by Associate Editor B. Chaib-Draa.

N. Lavrač and T. Urbančič are with Jožef Stefan Institute, 1000 Ljubljana, Slovenia and also with the University of Nova Gorica, SI-5000 Nova Gorica, Slovenia (e-mail: nada.lavrac@ijs.si; tanja.urbancic@ijs.si; stefan.bollhalter@ virtuelle-fabrik.com).

P. Ljubič, G. Papa, and M. Jermol are with Jožef Stefan Institute, 1000 Ljubljana, Slovenia (e-mail: peter.ljubic@ijs.si; gregor.papa@ijs.si; mitja. jermol@ijs.si).

S. Bollhalter is with Virtuelle Fabrik AG, CH-9320 Arbon, Switzerland.

Digital Object Identifier 10.1109/TSMCC.2006.889531 because it enables organizations' timely response to a new business opportunity, overcoming geographic dispersion of organizations and clients. In this correspondence, we use a broader term virtual organization (VO) instead of VE, as a VO can incorporate enterprises as well as other types of organizations. A VO can be formed dynamically from a cluster of organizations when a new business opportunity arises, and it is dissolved once the demand is fulfilled.

VO creation is a difficult process, requiring the strategic and management decision-making processes to be substantially different from those in traditional organizations. One of the key issues is appropriate VO partner selection as a response to a new business opportunity. Several criteria may be chosen, such as partners' quality, shortest time to market, cost issues, etc. To support these activities, a VO has to solve the problems of efficiently storing, updating, sharing, promoting, and transferring knowledge. In this effort, referred to as knowledge management [3], technological solutions, as well as organizational, economic, legislative, psychological, and cultural issues have to be addressed [4].

Similarly, for a new collaboration opportunity, a virtual team (VT) can be formed from a pool of individuals. Similar to the VE model for CNO, a VT model [5] can be utilized for the creation and operation of a dynamic team of experts willing to respond to a new collaboration challenge. The creation and operation of a VT is even more complex than is the collaboration in a VO, since a VT is a loose formation based on common interests of team members that are usually not collaborating based on formal contracts and obligations. The case becomes even more complex if a VT evolves from a community of experts that aim to fulfill a business opportunity. This implies several additional aspects to be considered, mainly related to the intellectual property rights (IPR) and a formal contract/agreement with a principal who offers a contract and who can be, at the same time, a member of the collaborating VT.

Recent research has shown that mutual trust can contribute to improved knowledge sharing, resource sharing, and taking joint risks. Trust brings many advantages to CNOs [6], [7], and should, therefore, be considered beforehand when creating a VO or a VT.

Trust is a very complex concept, dealing with trust in agents (individuals, organizations) and trust in information. According to many different views, ranging from sociology [8] to business [9], trust manifests itself in many different forms. In sociology, it is a relationship between actors that involves the suspension of disbelief that one actor will have toward another actor or idea. Personal meetings, regular communication, sharing of information and knowledge, and stable rules of the game may be sufficient means for trust management in moderately sized VOs/VTs. However, in large VOs/VTs, in which personal meetings and communication are hindered by the size of VO/VTs, it becomes indispensable to support trust modeling by (semi-)automated trust estimation and monitoring tools.

Existing agent-level trust models can be classified into three main categories [10]: 1) learning-based models using game theory approaches [11]; 2) reputation-based models aimed at social network and collaboration past performance modeling [12]; and 3) sociocognitivebased models aimed at assessing the outcomes of interactions between agents [13].

This correspondence presents an approach to modeling trust in collaborative networks of organizations and networks of individuals, using hierarchical multiattribute decision-support-based trust estimation. The approach is demonstrated in two scenarios - creating a network of collaborating organizations (VO) and a network of collaborating individuals (VT) - the first using a questionnaire-based approach and the second based on automated trust estimation through reputation and collaboration estimates computed from data gathered by Web crawling. These two scenarios originate from two European research projects, SolEuNet (2000-2003) and ECOLEAD (2004-2008). 
- The ECOLEAD project (http://www.ecolead.org) aimed at investigating the functioning principles of CNOs through establishing reference models and practical tools for creating and supporting VOs and VTs. The Virtuelle Fabrik (VF) cluster of enterprises in mechanical engineering, a CNO participating in the ECOLEAD project, serves as a case study for VO trust modeling in this correspondence.

- The SolEuNet project (http://soleunet.ijs.si) aimed at forming a VE of organizations with expertise in data mining [14] and decision support [15]. The complexity and difficulty of VO creation in this project partnership, due to partners' background in the (somewhat incompatible) business and academic worlds, has triggered the analysis of different VO organizational forms from the point-of-view of trust, and has enabled us to report on the lessons learned based on the principal-agent theory that was used for problem analysis in this case. Moreover, due to the prevailing scientific character of the consortium, the SolEuNet case study has also enabled us to perform an experiment in automated Webbased trust modeling, using simplified (scientific only) reputation and collaboration criteria measured on the data obtained by Web crawling. As scientific collaboration in the consortium had mainly the character of a VT activity, the experiment described in this correspondence deals with trust modeling in a network of individuals.

In the two case studies, we have based our approach on the data about partners' past performance and their reputation in the social network of collaborating partners. Therefore, we have focused on the reputation-based trust in line with a part of the FIRE integrated trust and reputation model [16]. For information gathering, we have used a simple questionnaire-based approach for VF in the VO case study, and Web crawling for SolEuNet in the VT case study. Note that this correspondence does not intend to provide a referential trust model, appropriate for different forms of VOs and VTs. It also does not list a comprehensive set of trust modeling criteria, e.g., as listed in one of the recent studies concerning VO breeding environments (VBEs) [17]. Instead, the aim of the correspondence is to propose a pragmatic approach to trust modeling, based on the data that can be realistically acquired either from network members themselves or from publicly available Web and database resources. The practical utility of the selected criteria, used for trust modeling using hierarchical multiattribute decision support methodology [15], is complemented by the visualization of the trust network, applicable for social network analysis purposes [18]. This theoretically sound methodology is further complemented by the principal-agent theory [19], which was used for in-depth analysis of trust-based organizational models considered in one of the two case studies.

The correspondence is organized as follows. Section II presents the background and related work in trust modeling. Section III presents the basics of hierarchical multiattribute decision support and social network analysis used as the main methodologies in the proposed trust modeling approach. Section IV outlines the principal-agent theory as a framework for analyzing the problem of trust between network partners, followed by the lessons learned from implementing three different organizational forms aimed at improved VO trust management, tested in the course of the SolEuNet project: 1) a fixed association of project partners; 2) a VE model with an exclusive marketing agent; and 3) a VE model involving several marketing agents. It also presents a simple questionnaire-based trust estimation method implemented in a decision support model, developed in the ECOLEAD project, that can be used to support VO creation. The approach is illustrated with a case study of modeling trust in the VF cluster of companies in mechanical engineering. In Section V, a Web-based trust modeling approach based on multiattribute decision support and social network analysis methodology using Web crawling and visualization technology is presented. The proposed approach has been applied to trust modeling in the SolEuNet partnership of individual experts. We conclude each case study with a reflection and lessons learned from the developed trust models. Sections VI and VII present the conclusions and further work.

\section{BACKGROUND AND RELATED WORK}

The concept of trust is related to the research in many disciplines ranging from economics and sociology to psychology and biology [20]. The main aspects of trust are trust to a partner, trust to information, and trust of itself. Reputation is a concept that is, usually, closely related to or merged with the concept of trust. There are many definitions of trust and reputation provided in [8], [9], and [20]-[22], some of which directly address VOs and VTs.

Development of trust models has been a topic of substantial research efforts. Several reputation models have been proposed by the multiagent community [23], where aggregated confidence and reputation information combine into a trust value. The SPORAS centralized [24] and the Histos decentralized model [24] were among the first trust models published. In SPORAS, trust values are shared between all partners by submitting all ranks to some centralized element. With the decentralized approach in Histos, the referee function was introduced to solve the problem of trust estimation in open communities. In the REGRET trust model [25], the model inputs are outcomes of all the partner interactions. Because some values are common and others known just to some partners, the model causes asymmetry in the trust network. FIRE is an integrated trust and reputation model that introduces uncertainty in the relations between partners [16]. The DIRECT reputation model was developed for the case of VO [22]. All values that are computed from the experience of a partner as well as from the mediated experience are stored locally at the partner. Some other activities can also be used for reputation assessment such as monitoring of the signals in the market, e.g., by analyzing reports in the media.

In a CNO, the notion of distributed trust is also very important. According to [26], distributed trust is "the representation of inputs to, and the process of making, trust decisions based on resources shared among multiple entities." The concept of distributed trust is important since it supports the local trust decisions based on shared trust resources.

Recent applications of knowledge technologies show their potential for improved trust modeling, knowledge and competence mapping, competence directory formation, agent selection and matching, as well as agent and competency search. Managing trust for the semantic Web also appears to be of particular interest of researchers [26], [27]. Some of these approaches can be used for trust modeling aimed at facilitating the agent-selection process and the matching of competencies for a new business opportunity.

Of special significance to our approach of evaluating trust (using a utility function) is the work of Richardson et al. [28]. Drawing the analogy with the semantic Web, different organizations in a network can be viewed as information sources (of varying qualities) and the competencies of each organization can be treated in a similar way to those of contents on the semantic Web in the form of logical assertions or statements. For each organization, which is directly linked to its partner, trust between them can be computed using a utility function like the ones proposed in this correspondence. Every organization also has a certain belief in its own statements asserted (which are basically its competencies). The belief of an organization in its partners' competencies is, then, computed and is used as a basic criterion for selecting partners when a new business opportunity arises. 
Social network analysis is an established research field aimed at modeling social network phenomena [29], [30]. Trust modeling research in social networks has also gained much attention. Recently, numerous networking tools have been made available to individuals and organizations mainly to help establishing and maintaining virtual communities. The common characteristic to all of them is that members build and maintain their own social networks based on trust, which are, then, connected to other networks through hubs (individuals that are members of two ore more networks).

The aim of our approach is to create a framework for trust modeling, enabling the creation of new trust models as instances of the proposed approach using hierarchical multiattribute decision support, social network analysis, and visualization.

\section{DECISION SUPPORT AND SOCIAL NETWORK ANALYSIS APPROACHES USED FOR TRUST MODELING}

In this correspondence, the term trust model is used to denote a reputation-based trust part of the FIRE trust model. It is focused on gathering ratings through a social network and on aggregating ratings based on the collaboration between partners/members of a collaborative network. The model is represented by a graph, where the size of nodes denotes reputation and arcs indicate collaboration between organizations/individuals that-in the case of CNOs-means collaboration in previous joint projects (see Figs. 3, 5, and 9). Visualization of the graph enables a useful insight into trust relationships, which can be used by a net-broker to support decision making in the process of $\mathrm{VO} / \mathrm{VT}$ creation for a new collaboration opportunity, and for improved trust estimation and management in existing VOs and VTs.

This section first introduces a decision support methodology that will be used for modeling trust in a network of collaborating organizations and collaborating individuals. It is followed by basic notions of social network analysis that allow for measuring the trust of individual network nodes by means of hub and authority weights.

\section{A. Hierarchical Multiattribute Decision Support}

Multicriteria decision analysis (MCDA) is a well-developed mathematical theory providing methods to cope with conflicting objectives [31], [32]. This correspondence suggests MCDA as the approach to support decisions related to trust modeling, restricted to the case of a single decision maker, whose ultimate task is to evaluate several alternatives, i.e., trustful VO configurations, with regard to multiple criteria. Naturally, the evaluation of alternatives leads to their ranking, which supports the selection of the most suitable one. Often, the decision criteria are structured in an attribute hierarchy, which decomposes the more general criteria into subcriteria.

For trust modeling, the decision-making problem of trust estimation can be decomposed into decision subproblems; e.g., a mutual trust estimate can be computed as a weighted sum of values of different subordinate decision criteria. This computation can be performed using utility aggregation functions, computing the values of top-level decision criteria by aggregating values of decision criteria at lower levels of a hierarchical decision tree. A hierarchical decomposition of a decision problem into subproblems is shown in Fig. 1 [32].

The value of a utility function is a function of weighted values of individual criteria. Weight elicitation methods for numerical MCDA models are numerous and well-developed, such as WSM, ELECTRE, TOPSIS [33], SMART, and SMARTER [34], and the like. A wellknown method in this field is the analytic hierarchy process (AHP) [35], in which weights are elicited by pairwise comparison of attributes. AHP is interesting for its ability to estimate the consistency of the assessment.

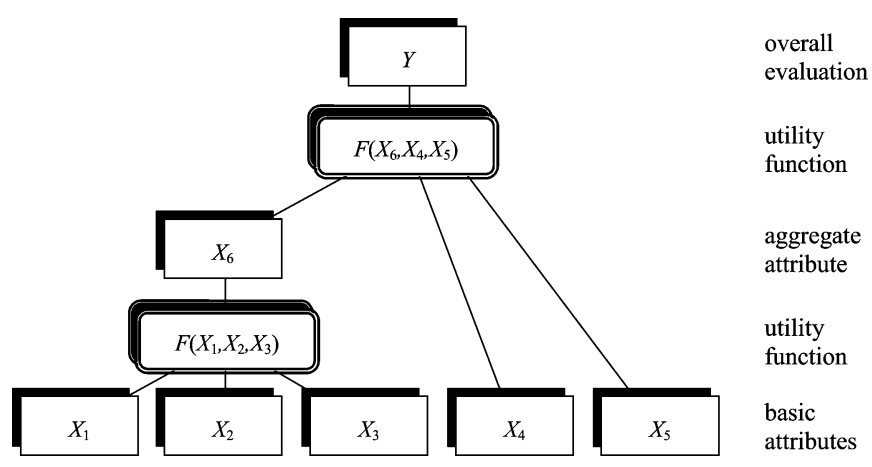

Fig. 1. Components of a multiattribute decision support model.

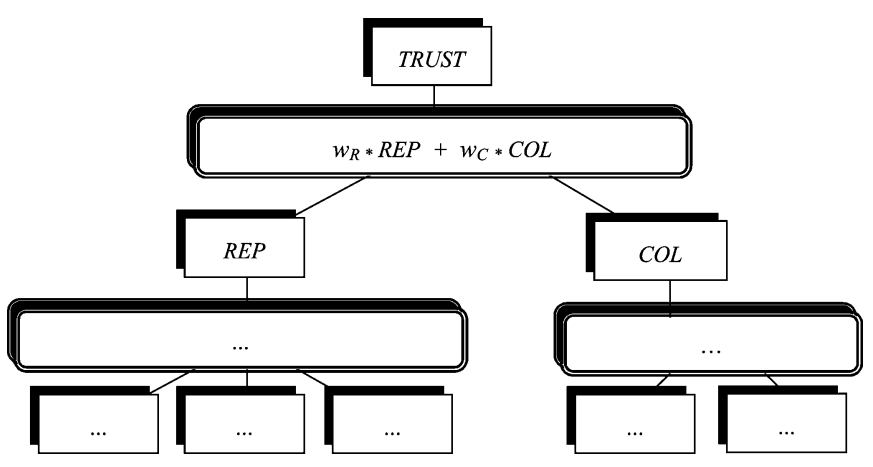

Fig. 2. Structure of a hierarchical multiattribute decision support model for trust estimation.

For qualitative (symbolic) multiattribute models, a suitable method is implemented in the system called DEXi [32], [36]. As opposed to systems like SMART that use numerical attributes and weights, DEXi uses qualitative attributes and rules. Use of qualitative attributes is suited for decision-making problems that are less structured and formalized [37]. Qualitative values are often denoted by words like "low," "appropriate," and "acceptable." Decision support system DEXi, used in our approach to trust modeling, is based on the DEX decision support tool [37] that is used to evaluate incompletely or inaccurately defined decision alternatives, by employing distributions of qualitative values, and evaluating them by methods based on probabilistic or fuzzy propagation of uncertainty.

Many factors affecting mutual trust need to be considered in a hierarchical tree of decision criteria to be used for trust modeling in a CNO. In this correspondence, we distinguish between individual node's attributes for estimating partner's reputation, and networking attributes (attributes describing relations between nodes) for estimating the performance in previous collaborations.

To compute a trust estimate, a utility function is, typically, defined as a weighted sum of different criteria. Such a utility aggregation function, typically used in hierarchical multiattribute decision support systems, is illustrated in Fig. 2 for the case of trust value estimation aggregated by computing a weighted sum of the reputation and collaboration estimates. A sample hierarchical decision tree in Fig. 2 illustrates how the values of top-level decisions can be computed by aggregating decisions at lower hierarchical levels of a tree of decision criteria.

When setting the weights of different criteria for trust estimate calculation, we have to consider a proper dependence between nodes, with higher weights assigned with more important criteria, and vice versa. Detailed evaluation of weights and their influence on the result (sensitivity analysis) are presented in Section IV-E. 


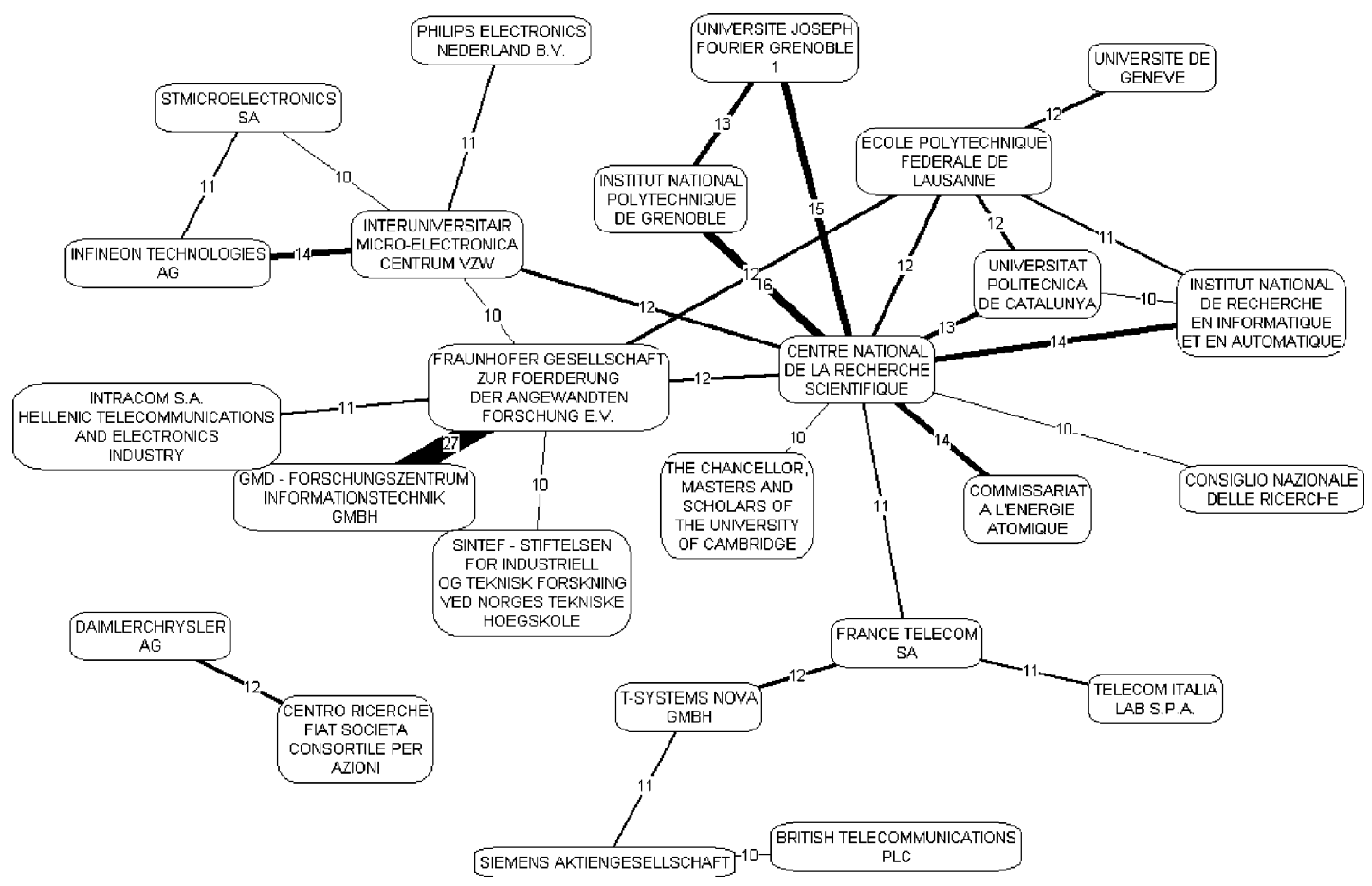

Fig. 3. Visualization of collaborations in European IST projects (taken from [38]). Organizations appearing in the graph had at least ten joint R\&D projects in the scope of the European 5th Framework 1st Programme.

1) Questionnaire-Based Trust Acquisition Approach: In a scenario of a small- or medium-sized CNOs, knowledge about mutual trust can be acquired through a simple questionnaire that a partner in a collaborative network can fill-in for a set of other partners of a CNO (individuals/organizations) with which it collaborated in past joint projects. As a member of the $\mathrm{CNO}$, every partner is required to provide this information that is stored in a CNO's organizational memory that partners of the $\mathrm{CNO}$ can access at any time. In this questionnaire-based scenario, a trust estimation model can be created through the following steps.

- Each partner is given a simple questionnaire in which it estimates its experience in past collaboration with other partners.

- For each questionnaire, numerical estimates of partner's reputation and past collaborations are computed by a multiattribute decision support system DEXi for each pair of agents.

- A graph of nodes (partners) and arcs (collaborations) is formed. The size of a node represents the estimated reputation of a network member, whereas the width of the arc represents the estimated collaboration between the connected partners. A sample graph is illustrated in Fig. 5.

2) Web-Based Trust Acquisition Approach: An alternative approach to trust modeling is through crawling of publicly available Web resources, e.g., as performed in the Project Intelligence toolbox [38]. Fig. 3 shows one of the Project Intelligence trust networks built from the data of partners of European 5th Framework IST projects. Nondirected links between partners represent mutual partners' trust through a simplified trust estimation measure: the number of joint collaborations in 5th Framework IST projects, used as the only connectivity/trust measure between the partners.

\section{B. Social Network Analysis}

The main goal of social network analysis is to detect and interpret patterns of social ties among actors [39]-[41]. In social networks, different aspects can be analyzed.
- Cohesion explores the detection of dense subnetworks of partners who "stick together," also known as cohesive subgroups (sometimes called "cliques," although—formally—a clique is a set of vertices that form a complete subgraph).

- Brokerage is concerned with the number and intensity of actor's connections, also known as its social capital. Some actors occupy central positions, showing their heavy involvement in the exchange and flow of information.

- Ranking, in contrast to the two previous cases, also takes into account the direction of connections-who is the initiator of a certain connection. This asymmetry in social relations points to social prestige and ranking.

In the two case studies described in this correspondence, the most interesting analysis is based on ranking of network partners, as $\mathrm{VO}$ and VT creation, basically, mean the selection of a subset of partners, and the goal is to choose a subset. In order to perform such a selection, partners must be ranked according to different criteria, including trust. Modeling trust using reputation and collaboration takes the following form (for simplicity, we have simplified the utility function by omitting the weights):

$$
\operatorname{TRUST}(X, Y)=\operatorname{REP}(Y)+\operatorname{COL}(X, Y)
$$

where $\operatorname{REP}(Y)$ and $\operatorname{COL}(X, Y)$ represent the reputation and collaboration, respectively. Collaboration of $X$ and $Y$ is mutual and can be seen as an undirected connection between two partners or as two directed connections bearing the same number (or weight). Adding reputation results in different weights of connections, thus making the network asymmetric. Note that partners with no collaboration (collaboration weight equals zero) estimate mutual trust based on reputation only. This makes our network a full graph, where every partner has some trust (a weighted connection) to all the other partners.

Ranking can take different approaches. It can be based on popularity, estimated by counting the incoming connections. In our case, this is 
not a good choice, since all partners have popularity $N-1$, given $N$ network partners. A better way is to sum the weights of incoming connections. Another approach is to remove all but a selected number (e.g., two or three) outgoing connections with the highest weights from each actor. In this way, each partner points just at few other most trustful partners. In this case, popularity can be calculated using only the count (sum) of incoming connections.

Another approach is to compute hub and authority values [18]. Nodes with most incoming links of high value represent the most trusted partners in the network (the authorities), while the nodes with most outgoing links represent the best connected actors (the hubs). Hubs and authorities exhibit what can be called a mutually reinforcing relationship, computed through iterative relaxation as a sum over $X$ such that there exists an edge from node $Y$ to node $X$ and from node $X$ to node $Y$, respectively

$$
\begin{aligned}
\operatorname{Hub}(Y) & =\sum_{Y \rightarrow X} \operatorname{Authority}(X) \\
\operatorname{Authority}(Y) & =\sum_{X \rightarrow Y} \operatorname{Hub}(X) .
\end{aligned}
$$

Again, hub and authority weights can be calculated on a full graph or on a reduced graph obtained by reducing the number of connections (e.g., two or three) as described earlier. This analysis gives us additional information: not only the popular actors are identified (as authorities), but also the actors surrounded by popular actors are identified (as hubs). Hubs and authorities, thus, provide additional node information and can be calculated in addition to the trust estimate based on reputation and collaborations, initially computed and visualized by the decision support approach.

\section{TRUST Modeling IN VOS: A QUESTIONNAIRE-BASED APPROACH}

This section first illustrates the problem of insufficient trust between network partners, as experienced in the SolEuNet project. The problem is explained by the principal-agent theory, which has helped the SolEuNet consortium to resolve the problem by gradually evolving the organizational forms, aiming at improved VO trust management. Lessons learned in this case study indicate that some of the problems could be resolved by eliminating information asymmetries between the partners, especially at the time of VO creation. This section presents a simple questionnaire-based trust estimation method, implemented in a decision support model developed in the ECOLEAD project. The approach is illustrated on a case study of modeling trust in VF, a Swiss cluster of companies in mechanical engineering.

\section{A. Principal-Agent Theory as a Framework for Trust Analysis}

The principal-agent theory [19], [42] is one of the main parts of a theory of economics that studies conflicting objectives and decentralized information. The principal-agent theory considers the problems of incomplete and asymmetric information in a situation when a task is delegated by a principal to a hired agent.

Two main actors of the partnership network, one investing financial capital and the other investing intellectual capital, establish the socalled principal-agent relationship [19]. The reason for starting this relationship is the inability of the principal to attain his goals by himself. The definition of who in the partnership is the principal and who is the agent may depend on a given business opportunity. Formally, the principal is the person/actor who is offering the contract. It is possible that the partner acting as the principal in one business opportunity will act as the agent in another. An essential characteristic of the principalagent relationship is that actions of the agent have consequences on the level of agent's welfare as well as on the utility of the principal.

The principal-agent theory points to problems arising from informational asymmetries between partners. Several types of informational asymmetries may arise: hidden characteristics, hidden action, hidden information, and hidden intentions. In the continuation, we briefly describe them and use them as elements of trust problem analysis.

- To make a network partnership effective and a project viable, partner relationships are regulated by a contract. The problem of hidden characteristics arises ex ante, before signing the contract, and refers to the possibility that the principal does not know all the relevant characteristics of the agent or his services. This information is obtainable only ex post. This phenomenon, known as adverse selection, may result in choosing an unsuitable partner.

- Hidden action and hidden information asymmetries come to force ex post, during the principal-agent relationship. Hidden actions occur when the principal is not able to directly monitor actions of the agent. Hidden information happens when the principal is able to observe actions of the agent but cannot judge their appropriateness because of the lack of specialized knowledge.

- Hidden intentions denote that the principal is aware of the opportunistic behavior of the agent but is unable to prevent it. This problem arises when the principal has made irreversible investments making him dependent on the agent. Costs related to such investment are called sunk costs. In the case of hidden intentions, the principal does not know the intentions of the agent ex ante. This phenomenon, known as the holdup situation, occurs when the agent exploits the principal's dependency on the agent.

The danger of information asymmetry can be diminished before concluding the contract, by signaling the agent's exceptional competences, screening of agent's characteristics, and agent's self-selection through contract formulation, which disables his own opportunistic behavior. An alternative way of dealing with asymmetric information is not in overcoming this asymmetry, but in trying to harmonize the interests of the agent with the interests of the principal by setting up suitable measures (for example, damage restitution and guarantees). The principalagent theory was conceived from the management perspective, helping the principal to identify and avoid agent's opportunistic behavior. If the theory were written from the agent's perspective, similar asymmetry could have been identified.

\section{B. Collaboration Models and the Lessons Learned}

Having gathered the core competencies and associated resources, a cluster of partner organizations has to choose a collaboration model enabling them to function as a business entity. This model should include protocols and standards for partner collaboration in distributed projects, protocols for information gathering, settled legal and IPR issues, as well as business plans. This section describes the collaboration models considered by the SolEuNet project partnership (Table I), their advantages, shortcomings, and lessons learned in terms of the principal-agent theory.

1) Fixed Association of Project Partners: About a year before the start of the SolEuNet project, four partners tried to form a fixed partner association, with one partner acting as an exclusive marketing agent. In this model, the problems of asymmetric distribution of information and the long-lasting process of building trust were prohibitive to the success of the partnership. The tendency of establishing fixed principal-agent relationships seemed to be of high priority to the marketing agent seeing 
TABLE I

SOLEuNet COLlaboration MODELS

\begin{tabular}{lll}
\hline \hline Type & Form & Net brokerage \\
\hline $\begin{array}{l}\text { Association of project partners } \\
\text { (Section IV-1) }\end{array}$ & Fixed & Single marketing agent \\
$\begin{array}{l}\text { VO model with a single marketing } \\
\text { agent (Section IV-2) }\end{array}$ & Flexible & $\begin{array}{l}\text { Single marketing agent } \\
\text { (net-broker) }\end{array}$ \\
$\begin{array}{l}\text { VO model with several marketing } \\
\text { agents (Section IV-3) }\end{array}$ & Most flexible & $\begin{array}{l}\text { Multiple marketing agents } \\
\text { (net-brokers) }\end{array}$ \\
\hline \hline
\end{tabular}

himself as the principal, while data mining and decision support expert partners, with established means of selling their own services, did not see themselves exclusively in the role of agents. The actors were, at that time, not aware of other possible forms of collaboration, or were not willing to establish them. The partner investing financial capital wanted to have fixed guarantees that other actors will be at his disposal for urgent tasks appearing in the market, and he, as the principal, would set the rules of the game. However, due to the lack of information and trust, it was impossible for data mining and decision support experts to accept these conditions and to give away their already established business relationships, as well as other ongoing and future business opportunities. With these two completely justified, but incompatible views of the involved partners, it does not come as a surprise that the attempt failed, despite long-lasting and difficult negotiations.

2) VO Model With an Exclusive Marketing Agent: The funding of the SolEuNet project provided a new opportunity for the collaboration of academic and business partners in the area of data mining and decision support. Twelve partners from seven countries (including the four partners from the attempt described earlier) entered the project. Clear goals were stated, and a detailed work plan was elaborated. The SolEuNet consortium decided to act as a VO, but also to set up a company in charge of marketing, while keeping the other VO partners for the knowledge-production function. By this decision, a two-actor network had been created: some partners investing mostly their intellectual capital, and the company investing mostly the financial capital. While some of the problems from the attempt with a fixed association were reduced, some others remained, mainly due to the different understanding of roles of each of the actors, amplified by the role of a net-broker acting as an exclusive marketing agent. Due to insufficient understanding of organizational and economic matters in a consortium with a prevailingly engineering background, both actors saw themselves as principals and the others as agents. This became clear only after analyzing the situation from the point-of-view of the principal-agent theory.

This approach also demonstrates the problem due to mixing of the two concepts: a VO and a VT concept, where the academic partners acting as individuals, rather than as representatives, of their organizations did not share the same business motivations with the business partners. Moreover, academic partners collaborated strongly in informal VTs, based on their strong research motivation.

The unsuccessful attempt to form VOs for business opportunities provided by the net-broker led to the development of a new VO model, involving distributed marketing by several net-brokers.

3) VO Model With Multiple Marketing Agents: In this model, the company that was initially intended to act as an exclusive VO netbroker is treated as one of the several potential net-brokers forming a net-brokerage network. An advantage of this solution was the distribution of marketing force, both geographically and socially. With distributed marketing points, there is also a better chance of responding to business opportunities. Moreover, if for some reason, one of the net-brokers preferred not to respond to a given business opportunity, it was able to pass such a request to other interested net-brokers, point- ing out the specific reasons for not responding itself. In the SolEuNet project, several net-brokers led to fewer tensions among partners, as dependencies were not so strong. In terms of the principal-agent theory, this model had the advantage that net-brokers and other partners were better motivated to prevent asymmetric distribution of information. Namely, as there were no long-term guarantees from any side, the involved parties were more aware of the importance of building trust as one of the preconditions for future collaboration. In particular, the danger of hidden intentions was reduced by introducing several possible marketing agents. Exploiting the principal's dependency on the agent is definitely not a good strategy in the situation where roles might easily be changed in the future. Building trust was recognized as one of the inevitable means of long-term success.

It also became clear that a trust evaluation model, like the one proposed in Section III, can improve the operation of a VO. In certain phases, especially early ones, it could also compensate for incomplete or asymmetric information. Unsuitable choice of an agent due to hidden characteristics is less likely if the principal has access to the other partners' trust evaluations of the agent based on their past collaborations. Also, since other partners may have specialized knowledge needed to evaluate and judge agent's actions (which the principle might not have), this can be reflected in their trust estimates made available to the principal for decision making.

The situation where any partner can act as a net-broker requires careful knowledge management that ensures updated information and its availability to all the partners. The SolEuNet consortium decided for knowledge and competence mapping, formation of a competence directory, and a knowledge base of completed projects, which all contributed to reducing the risk of hidden characteristics.

In this way, academic partners actually agreed to combine the VO and VT concepts into a successful SolEuNet CNO that, in addition, became aware of the importance of trust management as an important part of knowledge management.

\section{VF: A Questionnaire-Based Approach to Trust Modeling}

The approach to trust modeling, based on the DEXi hierarchical multiattribute decision support approach, was applied to modeling trust between partners of Swiss industrial cluster VF. A simplified trust model was built from questionnaire data about VF partners' reputation and collaborations.

In this case study, the VF net-broker provided his decision-making model as well as the data about the properties of each VF partner. Reputation was modeled through the following VF partner's attributes: activity, punctuality, reliability, partnership, risk willingness, and economical situation. Each of the properties had values from 1 to 6 (1: very bad, 6 : very good), and the overall reputation was computed as the average of values of the basic input attributes. Collaboration between VF partners was evaluated by values $0-3$ ( 0 : no collaboration, 1 : collaboration in two or less VOs per year, 2: collaboration in more than two VOs per year, 3: collaboration in more than five VOs per year), as shown in Table II.

The developed hierarchical decision support model for trust estimation is shown in Fig. 4. In the model, all the weights were initially set to 0.5 , while all the values were normalized to values on the $[0,1]$ interval as follows:

$$
\text { Normalized value }=\frac{\text { Actual value }- \text { Min value }}{\text { Max value }- \text { Min value }} .
$$

Fig. 5 shows the VF trust network. In the visualization tool developed for this application, an organization with greater trust is represented by a wider node and by a larger number of thicker arcs connecting the node with the collaborating partners. "Nontrustful" partners (below the 
TABLE II

(LEFT) INFORMATION ABOUT COMPANIES AND (RIGHT) THEIR COOPERATION

\begin{tabular}{|c|c|c|c|c|c|c|c|c|c|c|c|c|c|c|c|}
\hline \multirow[b]{2}{*}{$\Xi$} & \multicolumn{6}{|c|}{ reputation } & \multicolumn{9}{|c|}{ cooperation matrix } \\
\hline & 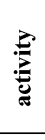 & 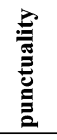 & 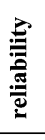 & 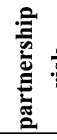 & 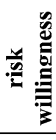 & 递 & 프 & 1 & 2 & 3 & 4 & 5 & 6 & 7 & $\ldots$ \\
\hline 1 & 2 & 5 & 6 & 4 & & 5 & 1 & & & & 3 & & & 1 & \\
\hline 2 & 4 & 5 & 6 & 5 & 3 & 5 & 2 & & & & & & & & \\
\hline 3 & 2 & 5 & 6 & 5 & 4 & 5 & 3 & & & & & & 1 & & \\
\hline 4 & 5 & 6 & 6 & 6 & 6 & 6 & 4 & 3 & & & & & 2 & 2 & \\
\hline 5 & 5 & 5 & 6 & 6 & 5 & 6 & 5 & & & & & & & 1 & \\
\hline 6 & 2 & 5 & 6 & 4 & 4 & 6 & 6 & & & 1 & 2 & & & & \\
\hline 7 & 5 & 5 & 6 & 6 & 6 & 4 & 7 & 1 & & & 2 & 1 & & & \\
\hline$\ldots$ & & & & & & & $\ldots$ & & & & & & & & \\
\hline
\end{tabular}
martix denote zero values, denoting no collaboration (zero values are omitted for clarity).

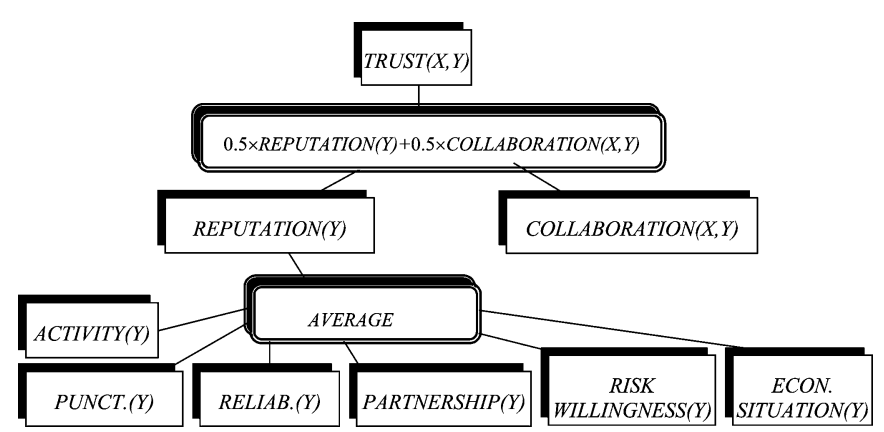

Fig. 4. Trust estimation model for VF.

user-defined threshold) do not appear as nodes of the trust network. In Fig. 5, reputation estimates are depicted by the size of nodes representing the companies, and the collaborations estimates by the width of arcs between the companies.

\section{VF Trust Modeling Lessons Learned}

The VF trust model, evaluated by the VF net-broker, resulted in the following lessons learned.

- The developed decision support approach and trust visualization tool are useful for VF trust estimation.

- The graph developed by the visualization mechanism shows an accurate picture of the status of VF and its potential VO configurations.

- Some known partner relationships were confirmed.

- Data collection is a very sensitive issue. There is a certain offending potential of collecting trust modeling data and graphically depicting partner relationships.

- Despite a potential danger of offending nonactive and nonconnected partners, the approach can also have the opposite, motivating effect: nonactive partners may explain the reasons for their situation and the desire for becoming more active members of the network.

\section{E. Sensitivity Analysis of Weights}

Fig. 6 presents the distribution of trust among the VF companies. As there are 66 companies, there are 4290 edges representing trust estimates between all the possible pairs of companies. When the

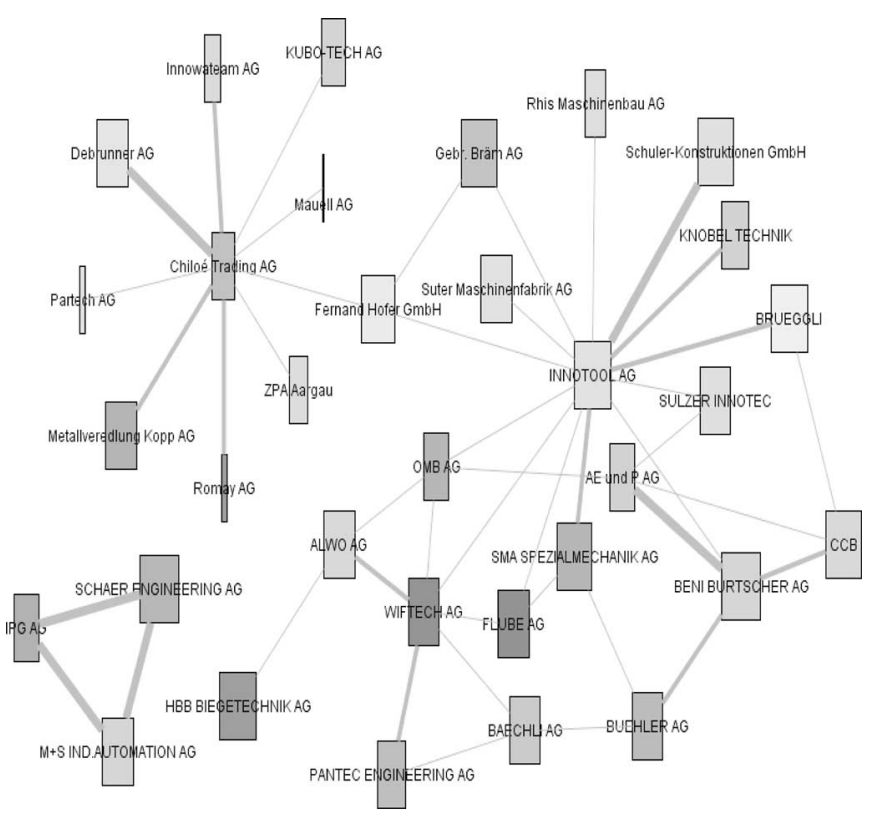

Fig. 5. Visualization of the VF trust network with node labels corresponding to company names. The figure shows the best connected subset of organizations of the VF industry cluster.

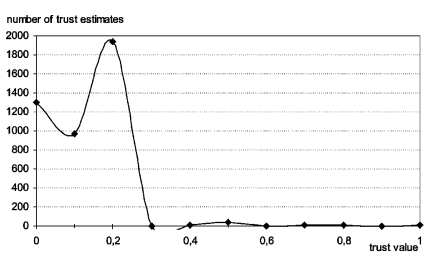

(a)

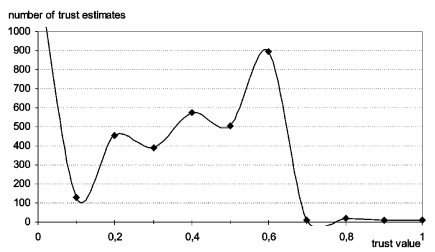

(c)

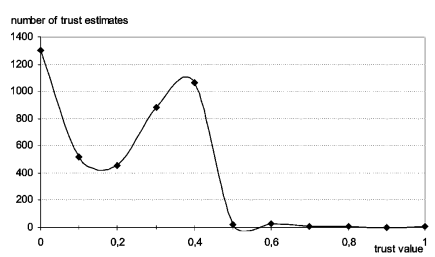

(b)

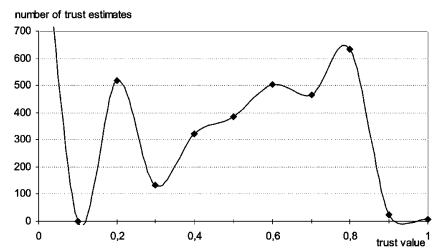

(d) number of trust estimates

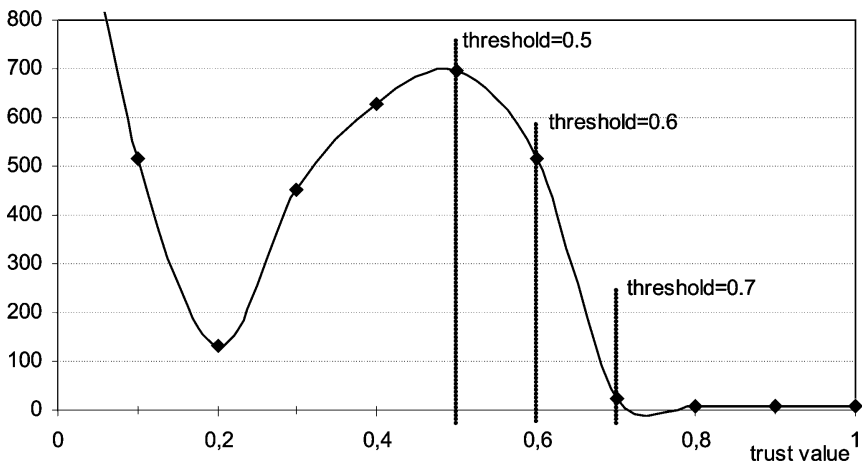

(e)

Fig. 6. Weights sensitivity analysis for trust calculation. (a) $w_{\mathrm{rep}}=0.2$ and $w_{\text {col }}=0.8$. (b) $w_{\text {rep }}=0.4$ and $w_{\text {col }}=0.6$. (c) $w_{\text {rep }}=0.6$ and $w_{\text {col }}=0.4$. (d) $w_{\text {rep }}=0.8$ and $w_{\text {col }}=0.2$. (e) $w_{\text {rep }}=0.55$ and $w_{\text {col }}=0.45$. 


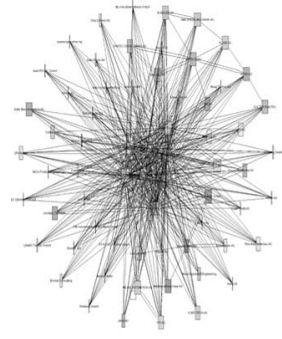

(a)

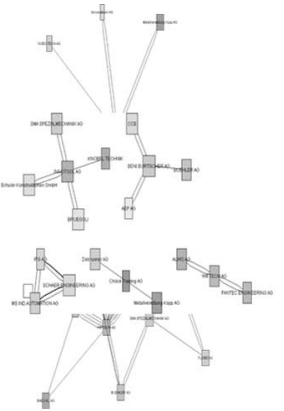

(b)

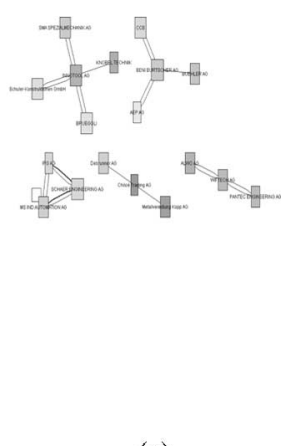

(c)
Fig. 7. Visualization of the VF trust network with different trust value thresholds. (a) 0.5. (b) 0.6. (c) 0.7 .

weight of reputation is set too low [Fig. 6(a)], the calculated trust estimates are mostly very low, and when the weight of reputation is too high [Fig. 6(d)], most of the trust estimates are very high. If the weights are set to 0.55 and 0.45 for reputation and collaboration, then the distribution of trust estimates can be approximated by a normal distribution [Fig. 6(e)] for a given case, where most of the trust estimates are in the middle of the range and the rest are almost equally distributed at the left- and right-hand sides. Some more trust estimates are at the lefthand side (lower), due to the lack of information about the reputation and/or collaboration for some companies; therefore, their trust estimate is equal to 0 (there are 1299 pairs with this trust value), or at least, very small. In other cases, one might need some other information to extract; therefore, weights should be set according to those needs.

The presented figures were used to find general-purpose weights, but when the search is oriented to some specifics, i.e., companies with high reputations or companies with many collaborations, then these weights can be set accordingly. In the case study, the default setting of weights to 0.5 for reputation and collaboration, thus, turned out to be appropriate.

Based on this analysis, we can get the threshold for searching the companies with a sufficiently high trust. In Fig. 7, three trust visualizations are presented. If the threshold is too low, the graph is very compact [Fig. 7(a)], while higher thresholds lead to thinner graphs [Fig. 7(b) and (c)] that provide a more insightful visualization of partners' trust relationship.

\section{Trust Modeling IN VTs: A Web-BAsed APPROACH}

A questionnaire-based approach, which was applied to modeling trust between partners of the VF industrial cluster, is a preferred means for the estimation of trust between organizations that have known each other from past collaborations. However, a computational Web-based trust modeling approach, similar to the one proposed in [43], is more adequate for roughly estimating the reputation and joint collaborations of partners (individuals or organizations) in newly established networks (at a VO or VT startup or in early stages of a new collaborative project) in the case that a consortium is build of numerous new partners whose past performance is not known in advance.

An approach to trust modeling investigated in the SolEuNet case study is through the analysis of publicly available Web resources. In this study, we concentrated on scientific trust estimation of a VT of experts collaborating in data mining and decision support scientific areas, measured through collaboration on research publications, as for each publication, a number of experts from SolEuNet formed a VT that collaborated until the challenge of publishing the result was fulfilled. Fig. 8 presents a model for trust estimation for each pair $(X, Y)$ of individuals collaborating in the SolEuNet project.

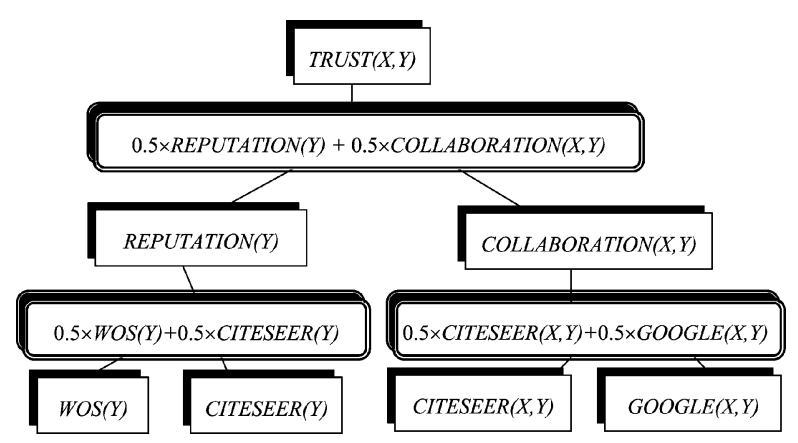

Fig. 8. Selected trust estimation model for the SolEuNet VT.

In order to estimate trust between the members of the SolEuNet scientific VT, the following procedure was applied.

- Collect the information about individuals' reputation based on the publication record of each individual member, using the information in the Web of Science and CiteSeer.

- Collect the information about past collaborations between each two individuals, using the information available in CiteSeer and using Google search.

- Calculate a trust estimate as a weighted sum of research reputation and joint collaborations estimates, using the TRUST $(X, Y)$ calculation function shown in Fig. 9 that is defined as follows:

$$
\begin{aligned}
\operatorname{TRUST}(X, Y)= & w_{R}\left(w_{\mathrm{Wos}} \operatorname{WOS}(Y)\right. \\
& \left.+w_{\mathrm{CiteCit}} \operatorname{CITESEER}(Y)\right) \\
& +w_{c}\left(w_{\mathrm{citeDoc}} \operatorname{CiTESEER}(X, Y)\right. \\
& \left.+w_{\text {Google }} \operatorname{GOOGLE}(X, Y)\right)
\end{aligned}
$$

where $w_{\mathrm{WOS}}, w_{\mathrm{CiteCit}}, w_{\mathrm{CiteDoc}}, w_{\text {Google }}, w_{R}$, and $w_{c}$ are the weights of Web of Science publications, CiteSeer citations, joint publications in CiteSeer, collaborations found by Google, and the overall reputation and collaborations weights, respectively. In the model used in the experiment, all the weights were set to 0.5 , while the numbers of publications, citations, joint publications, and collaborations were normalized to values on the $[0,1]$ interval. [Note that the functions used for trust estimation are not commutative, so trust of $X$ to $Y$ and trust of $Y$ to $X$ must be calculated. In the second case, the data for member $X(\operatorname{WOS}(X)$ and $\operatorname{CITESEER}(X))$ is used.]

- In the SolEuNet case study, in order to achieve more reliable estimates, Google search was replaced by coauthorship of chapters of the book in [44], as well as the search for coauthorship in the SolEuNet publications library (see the SolEuNet library at http://www.cs.bris.ac.uk/ SolEuNet/Tools/Reports/index.html).

Having calculated the trust estimates, one is able to rank individual researchers according to their research reputation, joint collaborations, and the overall trust estimate. The Web-based trust estimation model can also be used for other purposes: visualization of the entire trust network, as well as finding well-connected subgraphs with high trust estimates, representing "cliques" of individuals with strong mutual trust.

Fig. 9 shows Web-based estimates of research reputation and joint collaborations of 30 individuals participating in the SolEuNet consortium. The figure shows that project coordinators and workpackage coordinators were well linked due to many collaborations. Some welllinked individuals represent "cliques" of individuals, e.g., researchers from the same organization, typically, have more collaborations than do researchers from different organizations (the same color intensity is used to indicate the individuals' membership of the same organization). 


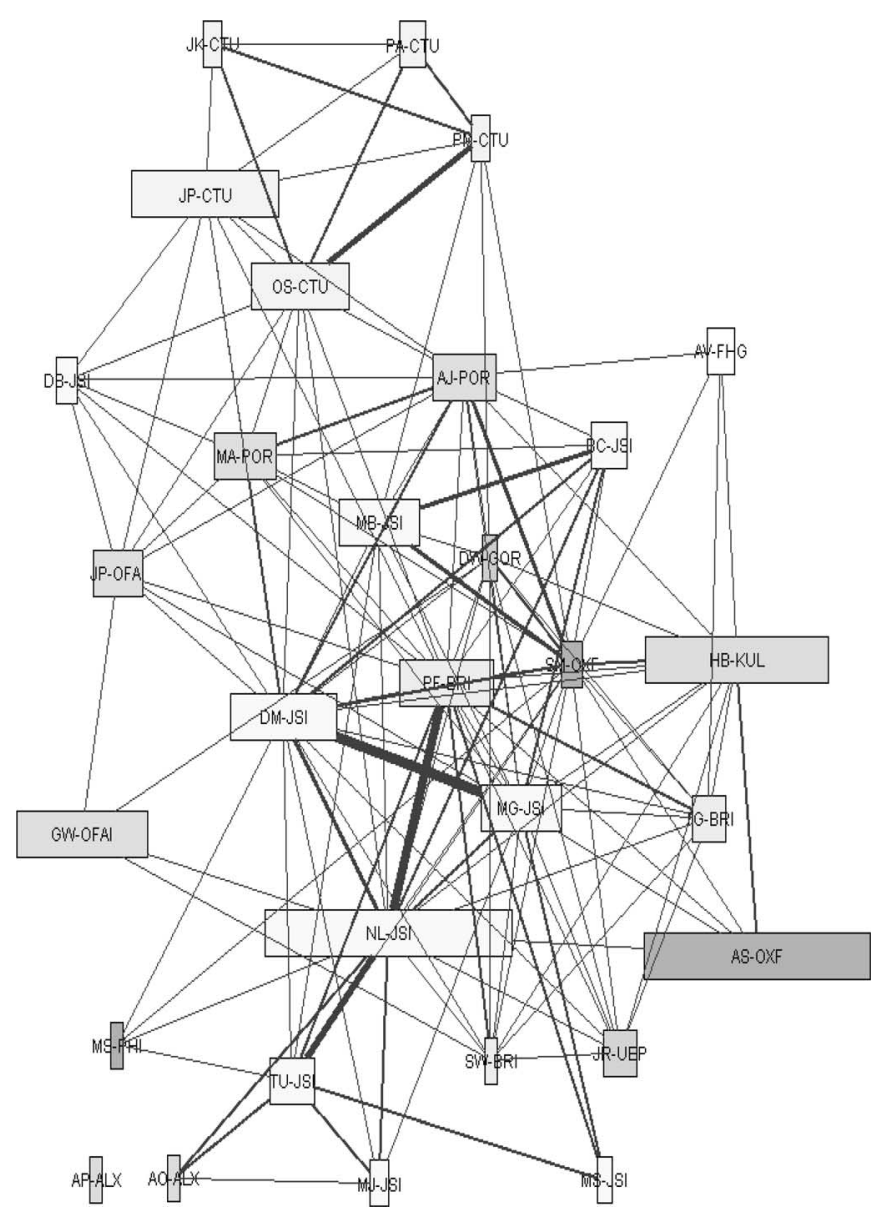

Fig. 9. A graph showing Web-based estimates of research reputation and joint collaborations of SolEuNet researchers. Research reputation estimates are depicted by the size of nodes, and the collaborations estimates by the width of arcs between two nodes.

TABLE III

POPULARITY OF SOLEUNET PARTNERS, AS WELl AS THEIR HUB AND AUTHORITY VALUES

\begin{tabular}{lcccc}
\hline \hline Actor & $\begin{array}{c}\text { Popularity } \\
\text { (sum) }\end{array}$ & $\begin{array}{c}\text { Popularity } \\
\text { (count) }\end{array}$ & $\begin{array}{c}\text { Authority } \\
\text { value }\end{array}$ & Hub value \\
\hline NL-JSI & 3.83 & 18 & 0.55 & 0.30 \\
DM-JSI & 2.40 & 4 & 0.35 & 0.32 \\
AS-OXF & 2.28 & 4 & 0.29 & 0.12 \\
HB-KUL & 2.06 & 4 & 0.26 & 0.14 \\
PF-BRI & 2.03 & 4 & 0.29 & 0.36 \\
$\ldots$ & $\ldots$ & $\ldots$ & $\ldots$ & $\ldots$ \\
\hline \hline
\end{tabular}

Using the concepts from social network analysis allows us to rank SolEuNet members according to their popularity, as well as hub and authority values, as shown in Table III. Fig. 10 shows the popularity, hub, and authority values of all SolEuNet actors. Note that the order of partners did not change much by using different ranking approaches, although the approach with summing up the values results in a more precise ranking.

\section{A. SolEuNet Trust Modeling Lessons Learned}

In the SolEuNet trust modeling application, the following lessons were learned.

- The developed visualization mechanism is very useful as the graph shows the status of SolEuNet VT past collaborations and its subgroup configurations.

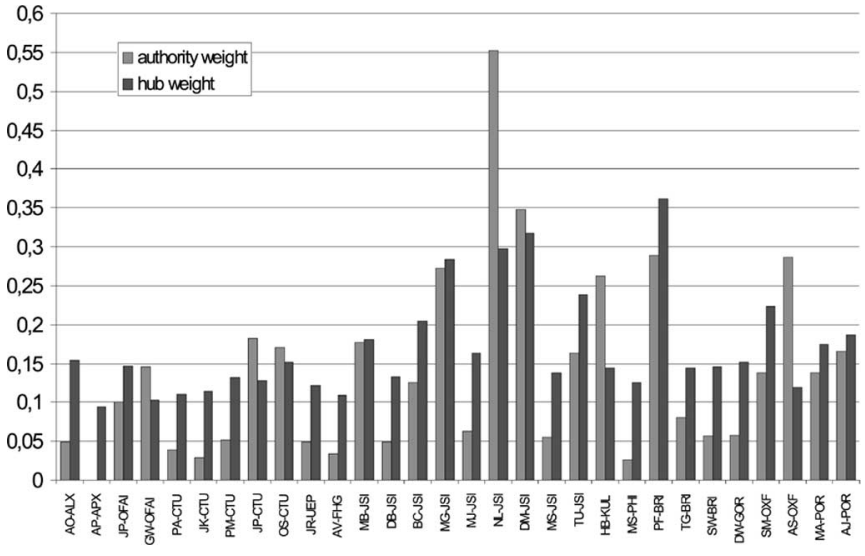

Fig. 10. Hub and authority values of all SolEuNet actors, when reputation and collaborations weights are set to 0.5 .

- The interpretation of results requests awareness of potentials traps of Web-based trust modeling, which may result in very rough and possibly erroneous approximations of trust among collaborating agents, using relatively unreliable estimates acquired from publicly available Web resources.

- Web-based data collection has to be performed carfully. First, Google search results in many useless/erroneous hits, as Google provides information on individuals with the same name, regardless of their profession. This can be partly avoided by including persons' affiliation in the query; however, affiliations may not be mentioned in the text, and/or many variants of the same institution name can occur (complete name in the original language, English name, only acronym, etc.). Moreover, as Google allows for maximum ten words to be searched at once, adding the affiliation name will disable having different variants of person names in the query (e.g., FirstName LastName AND FirstNameInitial. LastName AND ...). Therefore, in the final SolEuNet application, Google search was replaced by a more reliable search in the SolEuNet publications library.

- The proposed Web-based approach is especially well suited for modeling an unknown VT or VO environment. However, at that time, the developer, typically, does not have a sound basis for manual data cleaning to reduce the errors; e.g., detecting errors due to the occurrence of the same name denoting different people is impossible, or at least, extremely time-consuming. Moreover, there are limited publicly available data sources (mainly unstructured) about the past collaborations between companies in VOs.

- Data collection is a very sensitive issue. There is also a certain offending potential of trust estimation data and graphically depicting the relationships among network partners.

- Despite a potential danger of making mistakes due to erroneous data collection, offending less active and less connected actors, the approach can also have a motivating effect: the actors shown to be nonactive may either point to errors in data gathering, or explain the reasons for their actual situation and propose means for becoming more active members of the network in the future.

- For business trust modeling in networks of companies, different measures for calculating reputation and collaborations need to be considered than for the VT scientific trust estimation used in this case study. While whenever possible, a questionnaire-based approach should be used for scientific trust estimation, a Webbased approach may turn out to be very well suited as well, sparing the time needed for tedious data collection. 


\section{CONCLUSION}

This correspondence presents the experiences gained in the development of several organizational models for CNOs. The described models and the lessons learned, described in the principal-agent theory framework, may help find a suitable form of collaboration.

As VOs and VTs are forms of a voluntary partnerships, performing cooperative tasks and problem-solving based on the assumption of multilateral gain for all involved partners, deciding for several net-brokers in the SolEuNet business case is in alignment with this understanding of a CNO. Namely, if a net-broker should be given the same option of leaving at any time, having more than one net-broker makes a VO less vulnerable. In addition, partners are more motivated to build mutual trust since there are no long-term guarantees for their role.

The proposed approach to trust modeling can be used for decision support in the process of partner evaluation and selection when creating a VO or a VT for a new business opportunity, possibly serving several purposes:

- ranking of partners according to their reputation, joint collaborations, and the overall trust estimate;

- visualization of the entire trust network;

- finding well-connected subgraphs with high trust value, representing "cliques" of partners with high mutual trust.

In the two described cases, we implemented a pragmatic approach to trust modeling taking into account the data that can be gathered from partners in a VO (VF) or in a VT (SolEuNet) and the level of the automation of the complete process from data gathering to trust network visualization. It was shown that using the proposed approach for ranking, visualization of the trust network, and finding cliques of collaborating partners is relatively straightforward to implement.

\section{FURTHER WORK}

In the case studies, we have presented selected methods for ranking the network partners according to the trust they receive. Additional social-networks-based analysis can take cohesion and brokerage properties into the account. Using the brokerage measure, more centered partners might be preferred to marginal ones, while authority value calculation has taken into the account partners' centrality indirectly, since a partner cannot be an authority if his position is not central, or unless it receives a high trust from other authorities (which must be central). Cohesion could help us to first find dense groups, and then, the partners connecting two or more different partner groups.

In future work, we plan to further develop the trust modeling techniques, first initiated by the development of the Project Intelligence (http://pi.ijs.si) Web portal that enabled the analysis of IST projects of the European 5th Framework Programme [38]. The development of Project Intelligence was heavily based on text mining, Web mining, Web crawling, and graph visualization. These knowledge technologies have already shown their potential for modeling past collaborations and competencies of EU project partners, as well as partner ranking and partner matching for a new business opportunity, which was, in the case of FP5 IST project consortia, a call for new FP6 IST projects. Further developments are planned as part of the IST-World (http://www.istworld.org, 2005-2007) IST 6th Framework Programme Specific Support Action.

\section{REFERENCES}

[1] L. M. Camarinha Matos, H. Afsarmanesh, and R. Rabelo, "Supporting agility in virtual enterprises," in Proc. PRO-VE 2000-2nd IFIP Working Conf. Infrastruct. Virtual Enterprises, Florianopolis, Brazil, 2000, pp. 89104.
[2] L. M. Camarinha-Matos, H. Afsarmanesh, and R. Rabelo, Eds., E-Business and Virtual Enterprise: Managing Business-to-Business Cooperation. Norwell, MA: Kluwer, 2000.

[3] R. G. Smith and A. Farquhar, "The road ahead for knowledge management: An AI perspective," AI Mag., vol. 21, no. 4, pp. 17-40, 2000.

[4] J. McKenzie and C. van Winkelen, "Exploring E-collaboration space," presented at the Henley Knowledge Manage. Forum Conf., Henley on Thames, U.K., 2001.

[5] D. L. Pauleen, Virtual Teams: Projects, Protocols and Processes. Wellington, New Zealand: Victoria Univ. of Wellington, 2003.

[6] R. M. Morgan and A. D. Hunt, "The commitment-trust theory of relationship marketing," J. Marketing, vol. 58, no. 3, pp. 20-38, 1994.

[7] T. J. Norman, A. Preece, S. Chalmers, N. R. Jennings, M. Luck, V. D. Dang, T. D. Nguyen, V. Deora, J. Shao, W. A. Gray, and J. A. Fiddian, "Agentbased formation of virtual organizations," Knowl. Based Syst. J., vol. 17, no. 2, pp. 103-111, 2004.

[8] B. A. Misztal, Trust in Modern Societies. Cambridge, U.K.: Polity Press, 1998.

[9] J. Golbeck, B. Parsia, and J. Hendler, "Trust networks on the semantic web," presented at the Proc. Cooperative Inf. Agents, Helsinki, Finland, 2003.

[10] D. Sarvapali, D. H. Ramchurn, and N. R. Jennings, "Trust in multi-agent systems," Knowl. Eng. Rev., vol. 19, no. 1, pp. 1-25, 2004.

[11] M. Witkowski, A. Artikis, and J. Pitt, "Experiments in building experiential trust in a society of objective-trust based agents," in Trust in Cyber-Societies, R. Falcone, M. Singh, and Y. H. Tan, Eds. New York: Springer-Verlag, 2001, pp. 111-132.

[12] B. Yu and M. P. Singh, "An evidential model of reputation management," in Proc. 1st Int. Joint Conf. Autonomous Agents Multi-Agent Syst., vol. 1, C. Castelfranchi and L. Johnson, Eds., 2002, pp. 295-300.

[13] C. Castelfranchi and R. Falcone, "Social trust: A cognitive approach," in Trust and Deception in Virtual Societies, C. Castelfranchi and Y. H. Tan, Eds. Norwell, MA: Kluwer, 2000, pp. 55-90.

[14] J. Han and M. Kamber, Data Mining: Concepts and Techniques, 2nd ed. San Francisco, CA: Morgan Kaufmann, 2006.

[15] E. G. Mallach, Decision Support and Data Warehouse Systems. New York: McGraw-Hill, 2000.

[16] T. D. Huynh, N. R. Jennings, and N. R. Shadbolt, "Developing an integrated trust and reputation model for open multi-agent systems," in Proc. 7th Int. Workshop Trust Agent Soc., New York, 2004, pp. 65-74.

[17] S. S. Msanjila and H. Afsarmanesh, "Assessment and creation of trust in VBEs," in Network-Centric Collaboration and Supporting Frameworks, L. M. Camarinha-Matos, H. Afsarmanesh, and M. Ollus, Eds. New York: Springer-Verlag, 2006, pp. 161-172.

[18] J. M. Kleinberg, "Authoritative sources in a hyperlinked environment," in Proc. 9th ACM-SIAM Symp. Discrete Algorithms, New York, 1998, pp. 668-677.

[19] E. G. Furubotn and R. Richter, Institutions and Economic Theory: The Contribution of the New Institutional Economics. Ann Arbor, MI: Univ. of Michigan Press, 1997.

[20] D. Gambetta, Ed., Trust: Making and Breaking Cooperative Relations. Oxford, U.K.: Blackwell, 1990.

[21] N. Luhmann, Trust and Power. New York: Wiley, 1979.

[22] A. Avila-Rosas and M. Luck, "A direct reputation model for VOs formation," in Proc. 4th Int. Central Eastern Eur. Conf. Multi-Agent Syst. (CEEMAS 2005), Budapest, Hungary, pp. 460-469.

[23] S. Ramchum, N. R. Jennings, C. Sierra, and L. Godo, "Devising a trust model for multi-agent interactions using confidence and reputation," Appl. Artif. Intel., vol. 18, no. 9/10, pp. 833-852, 2004.

[24] G. Zacharia and P. Maes, "Trust management through reputation mechanisms," Appl. Artif. Intel. J., vol. 14, no. 9, pp. 881-908, Oct. 2000.

[25] J. Sabater and C. Sierra, "Social ReGreT, a reputation model based on social relations," ACM SIGecom Exc., vol. 3, no. 1, pp. 44-56, 2002.

[26] G. Mahoney. (2002). A Survey of Trust in Distributed Systems (Distributed Trust). Powerpoint presentation, [Online]. Available: http://www.panda. uvic.ca/seminars/storage/DistTrustSurvey.ppt

[27] P. Domingos and M. Richardson, "Mining the network value of customers," in Proc. 7th Int. Conf. Knowl. Discovery Data Mining, San Francisco, CA, 2001, pp. 57-66.

[28] M. Richardson, R. Agrawal, and P. Domingos, "Trust management for the Semantic Web," in Proc. 2nd Int. Semantic Web Conf., Sanibel Island, FL, 2003, pp. 351-368. 
[29] S. Wasserman and K. Faust, Social Network Analysis. Methods and Applications. Cambridge, U.K.: Cambridge Univ. Press, 1998.

[30] A. L. Barabási,Linked.The New Science of Networks. Cambridge, U.K.: Perseus, 2002.

[31] R. L. Keeney and H. Raiffa, Decisions With Multiple Objectives: Preferences and Value Tradeoffs. New York: Wiley, 1976.

[32] M. Bohanec, "Decision support," in Data Mining and Decision Support: Integration and Collaboration, D. Mladenić, N. Lavrač, M. Bohanec, and S. Moyle, Eds. Norwell, MA: Kluwer, 2003, pp. 23-35.

[33] E. Triantaphyllou, Multi-Criteria Decision Making Methods: A Comparative Study. Norwell, MA: Kluwer, 2000.

[34] W. Edwards and F. H. Barron, "SMARTS and SMARTER: Improved simple methods for multiattribute utility measurement," Org. Behav. Hum. Decis. Proc., vol. 60, pp. 306-325, 1994.

[35] T. L. Saaty, Multicriteria Decision Making: The Analytic Hierarchy Process. Pittsburgh, PA: RWS Publications, 1993.

[36] M. Bohanec. (2007). DEXL: A Program for Multi-Attribute Decision Making [Online]. Available: http://www-ai.ijs.si/MarkoBohanec/dexi.html

[37] M. Bohanec and V. Rajkovič, "DEX: An expert system shell for decision support," Sistemica, vol. 1, no. 1, pp. 145-157, 1990.
[38] M. Grobelnik and D. Mladenić, "Analysis of a database of research projects using text mining and link analysis," in Data Mining and Decision Support: Integration and Collaboration, D. Mladenić, N. Lavrač, M. Bohanec, and S. Moyle, Eds. Norwell, MA: Kluwer, 2003, pp. 157167.

[39] V. de Nooy, A. Mrvar, and V. Batagelj, Exploratory Social Network Analysis With Pajek.. $\quad$ Cambridge, U.K.: Cambridge Univ. Press, 2005.

[40] J. Scott, Social Network Analysis: A Handbook. London, U.K.: Sage, 2000.

[41] S. Wasserman and K. Faust, Social Network Analysis: Methods and Ap plications. Cambridge, U.K.: Cambridge Univ. Press, 1994.

[42] J. J. Laffont and D. Martimort, The Principal Agent Model: The Economic Theory of Incentives. Princeton, NJ: Princeton Univ. Press, 2002.

[43] Y. Matsuo, H. Tomobe, K. Hasida, and M. Ishizuka, "Finding social network for trust calculation," in Proc. 16th Eur. Conf. Artif. Intel., Valencia, Spain, 2004, pp. 510-514.

[44] D. Mladenić, N. Lavrač, M. Bohanec, and S. Moyle, Eds., Data Mining and Decision Support: Integration and Collaboration. Norwell, MA: Kluwer, 2003. 